resp. Subtraction eine vollkommen genaue Wägung auszuführen. Das Eintheilen des Halbkreises geschieht auf folgende Weise. Man belastet die eine Schale mit einem Zehnmilligramm-Stücke, bringt dann den Zeiger in eine solche Lage, dass sein in dieser Stellung wirkendes Gewicht gerade dem aufgelegten Gewichtsstücke das Gleichgewicht hält; dann legt man auf die Schale nur 9 Milligramm und markirt die dieser Belastung entsprechende Stellung des Zeigers auf dem Halbkreise. Auf diese Weise fährt man fort, indem man nach und nach jedesmal 1 Milligramm von der Schale wegnimmt bis $\mathrm{zu} 0 \mathrm{~d}$. h. bis der Zeiger in die Richtung des auf dem Wagebalken senkrechten Radius zurückgebracht ist. Jeder Theil dieser Graduirung kann dann in 10 Theile getheilt werden, deren jeder einer Belastung von $1 / 10$ Milligramm entspricht. Auf gleiche Weise verfährt man mit der andern Seite des Halbkreises, so dass sowohl der rechts, als der links liegende Quadrant einem Mehrgewicht von 1-10 Milligrammen entsprechend in Theile getheilt ist, welche dem Sinus des Abweichungswinkels des Zeigers proportional sind.

\title{
Asbestbad bei Destillationen in chemischen Laboratorien.
} Erlenmeyer empfiehlt*) bei Destillationen ron Flüssigkeiten, welche leicht stossen, sowie bei fractionirten Destillationen solcher Flüssigkeitsgemenge, welche einen hohen Siedepunkt haben, das Destillationsgefäss auf ein Drahtnètz zu stellen, auf welchem eine dünne, aber dichte Schicht kurzfaserigen, zu Stopfen doch nicht verwendbaren Asbestes ausgebreitet ist. Die auf solchem Asbestbade erhitzten Flüssigkeiten sieden nach Er l en me y e r ruhig und regelmässig und sind, wenn man die von der Flüssigkeit nicht berührten Gefässwände mit Asbest umgibt, was durch Anschmiegen des Drahtnetzes leicht geschehen kann, der Ueberhitzung viel weniger ausgesetzt als beim Erhitzen auf blossem Drahtnetze oder einem Sandbade.

Ueber die Construction eines Vaporimeters **). Die Schwierigkeiten, welche sich der genanen Bestimmung des Siedepunktes concentrationsfähiger Flüssigkeiten z. B. von Salzlösungen entgegenstellen, haben G. Th. Gerlach veranlasst in dieser Richtung Versuche anzustellen, deren Resultat er in der Monatschrift des

*) Zeitschrift f. Chem. u. Pharm. 1864, p. 639.

**) Monatschrift, des Gewerberereins zu Köln 1864. Heft 6. p. 161. 
Cölner Gewerbevereins veröffentlicht. Das von ihm empfohlene Verfahren versucht zur Erreichung des Zweckes die Temperatur zu ermitteln, bei welcher die Spannkraft der aus diesen Lösungen entwickelten Dämpfe dem Druck einer Atmosphäre das Gleichgewicht hält. Als leitende Idee diente dem Verfasser die Construction des Vaporimeters von Geissler in Bonn, welches aus der Spannung der Alkoholdämpfe bei der Siedetemperatur des Wassers die Concentration des Weingeistes schnell ermitteln lässt.

Die Beschreibung des in allen seinen Theilen durchsichtigen Apparates gebe ich unter Hinweisung auf die beigegebene Abbildung auf Tafel II..Fig. II u. III mit des Verfassers eigenen Worten:

$a b c d$ ist ein Gefäss von Metallblech, welches bei e eine Oeffnung hat, die mit einem durchbohrten Gummistopfen verschlossen wird. Durch die Oeffnung dieses Stopfens geht ein zweimal rechtwinkelig gebogenes Glasrohr, dessen kürzeres Ende so ausgezogen ist, dass es in einem durchbohrten Gummistopfen befestigt werden kann. In ein Fläsehchen $\mathrm{f}$ Fig. 2 kommt die zu untersuchende Flüssigkeit und alsdann Quecksilber bis ungefähr zar Marke g. Der durchbohrte Gummistopfen $\mathrm{h}$ wird jetzt in das Glas $\mathrm{f}$ eingesetzt, nachher die Glasröhre in den Gummistopfen $h$ befestigt; hierauf wird der Apparat in die umgekehrte Lage gebracht, wie es Figur 3 zeigt. Durch diese Umkehrung kommt das Quecksilber wieder nach unten und über dem Quecksilber befindet sich die zu untersuchende Flüssigkeit. Nachdem der Apparat so weit vorgerichtet ist, wird über das Gefäss $a b c d$ ein Gummiring i $\mathrm{k}$ gestülpt, der zur Aufnahme eines Glascylinders (eines gewöhnlichen Lampenglases) dient.

Das Gefäss von Metallblech und der Cylinder werden jetzt mit Chlorcalciumlösung angefüllt, so weit, dass diese Lösung das Fläschchen f um mehrere Zoll übersteigt. Da Chlorcalciumlösung weit über $100^{\circ} \mathrm{C}$. erhitzt werden kann, ehe sie zum Sieden kommt, so ist sie sehr gut zu einem erwärmenden Bade zu verwenden. Der ganze Apparat wird jetzt auf einem Dreifuss über der Gasflamme erhitzt. In Folge der Erwärmung dehnt sich anfangs Quecksilber und Flüssigkeit aus, und das Quecksilber dringt in Folge dessen in das Glasrohr ein. Bei einer engen Röhre kann leicht der Fall eintreten, dass allein in Folge der Ausdehnung der Flüssigkeit das Quecksilber bis zum Punkte 1 steigt; es ist deshalb nöthig, möglichst weit unterhalb dieses Punktes eine Kugel in die Röhre zu blasen, oder durch Biegen der Röhre (wie es die punktirte Linie anzeigt) eine Verlängerung derselben 
zu bewerkstelligen, da es ja bei diesen Versuchen in der Absicht liegt, aus der Flüssigkeit etwas Wasserdampf zu entwickeln, ohne dass der Druck einer Queksilbersäule diesen Vorgang hindere.

Aus dem Gesetze der Hydrostatik geht hervor, dass das Quecksilber sowohl im Fläschchen, als in dem Schenkel der Glasröhre gleich hoch stehen muss, wenn auf beiden Seiten derselbe Druck stattfindet. Der Druck auf das Quecksilber in der Glasröhre ist bekannt, es ist der Druck der Atmosphäre, der dem jedesmaligen Barometerstand entspricht. Ist also der Stand des Quecksilbers in dem Fläschchen $\mathbf{f}$ und in der Glasröhre gleich hoch, so geht daraus hervor, dass der gebildete Wasserdampf aus der. F'lüssigkeit die Spannung habe, um dem Drucke einer Atmosphäre das Gleichgewicht zu halten, oder mit anderen Worten, dass die Temperatur des Dampfes diejenize des Siedepunktes der Flüssigkeit sei. Die Temperatur des Dampfes lässt sich aber aus der Temperatur des Chlorcalciumbades ermitteln.

In der Construction des Apparates liegt es, dass in Folge der Füllung sich jederzeit über dem Quecksilber in der Röhre etwas von der Lösung befindet, womit das Fläschchen $\mathrm{f}$ gefüllt wurde; natürlich übt die Höhe dieser Flüssigk itsschicht einen Druck auf den gebildeten Wasserdampf aus, und zwar lässt sich dieser Druck leicht berechnen und in dem Drucke einer Quecksilbersäule ausdrücken, wenn man die Höhe dieser Flüssigkeitsschicht mit dem specifischen Gewichte der Flüssigkeit multiplicirt und durch das specifische Gewicht des Quecksilbers dividirt. Um in dieser Beziehung lästige Correctionen zu ersparen, setze ich auf das Ende der Glasröhre einen Gummischlauch $m$ and sauge die überstehende Flüssigkeit ab. Es thut der Richtigkeit des Versuches gar keinen Abbruch, wenn man bei dieser Gelegenheit etwas Quecksilber mit enfernt; die einzige Folge hierron würde sein, dass sich etwas mehr Dampf bilden kann; die Menge des Dampfes ist aber unter diesen Umständen ganz unabhängig von dessen Spannung. Nach der Entfernung dieser überstehenden Flüssigkeit setze ich das Kugelrohr n auf den Gummischlauch, um bei unvorsichtiger Erwärmung des Chlorcalciumbades als Behälter für das aufsteigende Quecksilber zu dienen. Dieser Apparat liesse sich in gebührend veränderter Form natürlich auch zur Bestimmung von Siedepunkten bei erhöhtem Drucke rerwenden, je $760 \mathrm{Mm}$. höherer Quecksilberstand in der Röhre entsprechen einer Atmosphäre Ueberdruck; namentlich aber dürfte der Apparat ein praktisches Instrument sein, Siedepunkte von Flüssigkeiten zu bestimmen, von denen nur wenige Tropfen zu Gebote stehen. 
Gerlach bemerkt ferner, dass sich wie stets beim Erwärmen von Flüssigkeiten, die von einer kältern Atmosphäre umgeben sind, auch hier die unangenehme Wirkung der Strömungen in der Chlorcaliumlösung bemerkbar mache, namentlich beim Abkühlen des Bades die Quecksilbersäule oft sehr plötzlich sinke, daher ein vorsichtiges Reguliren der erwärmenden Flamme sowie Umrühren des Bades nöthig sei.

Wenn auch der Einfluss der Flüssigkeitsschichte im Fläschchen, welche nicht in Dampfform verwandelt wurde, ein äusserst geringer ist, so darf er theoretisch doch nicht vernachlässigt werden; für je 13,5 Mm. unverdampftes Wasser im Fläschchen wird das Quecksilber in der Röhre $1 \mathrm{Mm}$. höher steigen müssen als das Quecksilber im Fläschchen, weil das specifische Gewicht des Quecksilbers 13,5 Mal so gross ist, als das des Wassers. In der Zeichnung ist dieser Punkt mit Null bezeichnet.

Schliesslich macht Gerlach noch darauf aufmerksam, dass dieser einfache Apparat wohl noch mancher Verbesserung fähig sei, doch habe er sich nit der Mittheilung derjenigen Form begnügen wollen, in welcher er den Apparat zusammengestellt habe; eine Tabelle, enthaltend Siedepunktsbestimungen von Salzlösungen folgt dann noch als Anhang, doch muss auf die Wiedergabe dieser Zahlen hier verzichtet und in Bezug hierauf auf die Originalabhandlung verwiesen werden.

Zur Spectralanalyse. In den Abhandlungen der naturfor schenden Gesellschaft zu Halle, Bd. IX., veröffentlichte Brassack" eine grössere Arbeit unter dem Titel, Spectralanalytische Untersuchungen der Metalle", und ist dieselbe bereits im Buchhandel als Abdruck erschienen. Da diese Arbeit manches interessante Neue bringt, so sollen in Nachfolgendem die wesentlichsten Resultate, welche Brassack erhalten hat, in gedrängter Kürze mitgetheilt werden.

Zur Darstellung der Spectra schlug Brassack denselben Weg ein, welchen schon Masson*) betreten hat. Ein Ruhmkorff'scher Inductionsapparat, der zur Verstärkung mit einer Leydener Flasche von grossem Belege verbunden und durch zwei mässig grosse Bunsen'sche Elemente in Thätigkeit gesetzt war, diente zur Hervorbringung des Funkenstromes, welcher continuirlich zwischen Elektroden

*) Annales de chim. et de phys. T. 31 ; p. 295. 